\title{
Effects of pre-stretch, compressibility and material constitution on the period-doubling secondary bifurcation of a film/substrate bilayer
}

\author{
Z.X. Cai ${ }^{\mathrm{a}}$, Y.B. Fu ${ }^{\mathrm{b}, *}$ \\ ${ }^{a}$ Department of Mechanics, Tianjin University, Tianjin 300072, China \\ ${ }^{b}$ School of Computing and Mathematics, Keele University, Staffordshire ST5 5BG, UK
}

\begin{abstract}
We refine a previously proposed semi-analytical method, and use it to study the effects of pre-stretch, compressibility and material constitution on the period-doubling secondary bifurcation of a uni-axially compressed film/substrate bilayer structure. It is found that compared with the case of incompressible neo-Hookean materials for which the critical strain is approximately 0.17 when the thin layer is much stiffer than the substrate, the critical strain when the Gent materials are used is a monotonically increasing function of the constant $J_{m}$ that characterizes material extensibility, becoming as small as 0.12 when $J_{m}$ is equal to 1 , whereas for compressible neo-Hookean materials the critical strain is a monotonically decreasing function of Poisson's ratio; the period-doubling secondary bifurcation seems to become impossible when Poisson's ratio is approximately equal to 0.307 . The latter result may indicate that when Poisson's ratio is small enough there are other preferred secondary bifurcations - an example is given where a secondary bifurcation mode with $7 / 4$ times the original period occurs at a lower strain value. The effect of a pre-stretch (compression or extension) in the substrate is not monotonic, giving rise to a critical strain that varies between 0.15 and 0.22 .
\end{abstract}

Keywords: thin-film/substrate bilayer, wrinkling, period-doubling, bifurcation, nonlinear elasticity.

\section{Introduction}

Stress-induced pattern formation in soft materials at the micrometer and submicrometer scales is now well recognized to have a wide range of applications ranging from cell patterning [1], optical gratings [2-4], and creation of surfaces with desired wetting and adhesion properties [5-7], to the deduction of material properties of ultrathin films [8, 9]. We refer to Bowden et al. [10, 11], Li et al. [12], the book by Goriely [13], and the more recent papers by Wang and Zhao [14] and Holland et al.

${ }^{*}$ Corresponding author at: School of Computing and Mathematics, Keele University, Staffordshire ST5 5BG, UK

Email address: y.fu@keele.ac.uk (Y.B. Fu) 
[15] for a comprehensive review of the literature and applications. As a prototypical structure, the buckling and post-buckling of a thin-film/substrate bilayer structure has probably received the most attention, partly because of the variety of behaviors that it exhibited and their obvious relevance to more complicated structures [1634]. If both the layer and substrate are composed of neo-Hookean materials with shear moduli $\mu_{f}$ and $\mu_{s}$, respectively, then there exists a critical moduli ratio which is approximately equal to 1.74 such that the initial wrinkling bifurcation is supercritical when $\mu_{f} / \mu_{s}>1.74$ and subcritical otherwise [35,36]. It can then be expected, and indeed confirmed by many recent numerical and experimental studies, that in the subcritical regime localization is the norm $[37,38]$ whereas in the supercritical regime period-doubling secondary bifurcation is the norm [39, 40].

This paper is a sequel to our earlier paper Fu and Cai [41] where attention is focused on the supercritical regime and the exact nonlinear elasticity theory combined with an asymptotic perturbation procedure is employed to derive the critical stretch value at which period-doubling secondary bifurcation takes place. The derived results agreed well with the numerical simulation and experimental results of Cao and Hutchinson [42] and Brau et al. [43], and the proposed approach complements earlier analytical studies by Brau et al. [43], Zhao et al. [44], and Zhuo and Zhang $[45,46]$ that are more approximate in nature. It was pointed out towards the end of $\mathrm{Fu}$ and Cai [41] that the proposed methodology could deal with any material constitution (compressible or incompressible, neo-Hookean or otherwise) and arbitrary pres-stretch in the substrate. This is now verified in the present paper.

When the materials are incompressible, the formulation is different from the case when the materials are compressible because of the introduction of a Lagrange multiplier and consideration of the incompressibility condition. To make our presentation as concise as possible, we shall not deal with the incompressible case separately, but rather view it as the limit of the compressible case when Poisson's ratio tends to $1 / 2$. Thus, using a single formulation we shall consider a strain-energy function given by

$$
W=-\frac{\mu}{2} J_{m} \log \left(1-\frac{I_{1}-3}{J_{m}}\right)-\mu \log J+\frac{\mu^{*}}{2}(J-1)^{2},
$$

where $J=\operatorname{det} \boldsymbol{F}, \boldsymbol{F}$ being the deformation gradient, $I_{1}$ is the first principal invariant of $\boldsymbol{F}^{T} \boldsymbol{F}, \mu$ is the ground-state shear modulus, $J_{m}$ is a material constant characterizing material extensibility, and $\mu^{*}$ is a constant that is related to Poisson's ratio $\nu$ through $\mu^{*}=2 \mu \nu /(1-2 \nu)$. The above strain energy function may be referred to as a compressible Gent material model. Under the double limit $\mu^{*} \rightarrow \infty, J \rightarrow 1$ such that $\mu^{*}(J-1)$ remains finite, it reduces to the incompressible Gent material model, which, under the further limit $J_{m} \rightarrow \infty$, recovers the classical neo-Hookean material model. On the other hand, by taking the limit $J_{m} \rightarrow \infty$, it reduces to the compressible neo-Hookean material model that has been used in many studies to assess the effects of compressibility.

The rest of this paper is divided into five sections as follows. The next two sections are devoted to the first bifurcation and are concerned with the linear analysis and 
post-buckling solutions, respectively. The (weakly nonlinear) post-buckling solutions can be obtained to any order in terms of a small parameter that characterizes the departure of the compressive strain from its critical value. This asymptotic solution is expected to provide a good approximation to the exact bifurcation solution for values of this parameter up to around 0.6. As this parameter is increased, a period-doubling secondary bifurcation will take place when the parameter reaches a critical value. The latter critical value is determined by a linearized bifurcation problem containing coefficients that are periodic functions because the post-buckling solution mentioned above is periodic. Thus, it has a similar structure to the classical Mathiew equation with the spatial variable along the interface here playing the role of time, and the secondary bifurcation is simply a subharmonic resonance phenomenon. The analysis is conducted in Section 4. Some numerical results are presented and compared with those available in the existing literature. The paper is concluded with a summary and some additional comments.

\section{First bifurcation - linear analysis}

We first summarize the incremental governing equations that are valid for both the first and secondary bifurcations. To this end, we consider a general hyperelastic body $B$ that possesses an initial unstressed configuration $B_{0}$. A static deformation (homogeneous or inhomogeneous) is applied to $B_{0}$ to produce a finitely stressed equilibrium configuration denoted by $B_{e}$. In order to determine whether $B_{e}$ may suffer a bifurcation or not, we superimpose on $B_{e}$ a small amplitude displacement, and the resulting configuration, termed the current configuration, is denoted by $B_{t}$. The position vectors of a representative particle relative to a common rectangular coordinate system are denoted by $\boldsymbol{X}, \boldsymbol{x}$ and $\tilde{\boldsymbol{x}}$, with associated coordinates $X_{A}, x_{i}$ and $\tilde{x}_{i}$ in $B_{0}, B_{e}$ and $B_{t}$, respectively. We write

$$
\tilde{\boldsymbol{x}}=\boldsymbol{x}+\boldsymbol{u}(\boldsymbol{x}),
$$

where $\boldsymbol{u}(\boldsymbol{x})$ is a small-amplitude displacement associated with the incremental deformation $B_{e} \rightarrow B_{t}$. Throughout this paper we employ the summation convention, and use e.g. $u_{i, A}$ and $u_{i, j}$ to denote $\partial u_{i} / \partial X_{A}$ and $\partial u_{i} / d x_{j}$, respectively.

We define the incremental stress tensor $\chi_{i j}$ through

$$
\chi_{i j}=\bar{J}^{-1}\left(S_{A i}-\bar{S}_{A i}\right) \bar{F}_{j A},
$$

where $\overline{\boldsymbol{F}}$ is the deformation gradient corresponding to the deformation $B_{0} \rightarrow B_{e}$, $\bar{J}=\operatorname{det} \overline{\boldsymbol{F}}$, and $\bar{S}_{A i}$ and $S_{A i}\left(=\partial W / \partial F_{i A}\right)$ are the nominal stress associated with the deformations $B_{0} \rightarrow B_{e}$ and $B_{0} \rightarrow B_{t}$, respectively. It follows from the identity $\left(\bar{J}^{-1} \bar{F}_{j A}\right)_{, j}=0$ that the equilibrium equation $S_{A i, A}=0$ can be reduced to

$$
\chi_{i j, j}=0,
$$

whereas a series expansion of the right hand side of (2.2) yields

$$
\chi_{i j}=\mathcal{A}_{j i l k}^{1} u_{k, l}, \quad \mathcal{A}_{j i l k}^{1}=\left.\bar{J}^{-1} \bar{F}_{j A} \bar{F}_{l B} \frac{\partial^{2} W}{\partial F_{i A} \partial F_{k B}}\right|_{\mathbf{F}=\overline{\mathbf{F}}},
$$


where $\mathcal{A}_{\text {jilk }}^{1}$ are the components of the first-order tensor of instantaneous elastic moduli in $B_{e}$ [47] and the expression for $\chi_{i j}$ has been linearized (the nonlinear terms will be restored in the next section).

We now specialize to the case when the finitely deformed configuration $B_{e}$ consists of a coated half-space (or a film/substrate bilayer) that is subjected to a uniaxial compression, with the half-space and coating corresponding to $-\infty \leq x_{2} \leq 0$ and $0 \leq x_{2} \leq h$, and having material constants $\left(\mu, \nu, \mu^{*}, J_{m}\right)$ and $\left(\hat{\mu}, \hat{\nu}, \hat{\mu}^{*}, \hat{J}_{m}\right)$, respectively. Correspondingly, all the field variables associated with the coating will be distinguished by a superimposed hat as well. Thus, the governing equations (2.3) and (2.4) apply to the region $-\infty \leq x_{2} \leq 0$, but for the coating we have instead

$$
\hat{\chi}_{i j}=\hat{\mathcal{A}}_{j i l k}^{1} \hat{u}_{k, l}, \quad \hat{\chi}_{i j, j}=0,
$$

which from now on will be referred to as $\left(2.3^{*}\right)$ and $\left(2.4^{*}\right)$ with the stars signifying the fact that they are the counterparts of (2.3) and (2.4) appropriate to the thin layer. This convention concerning the star will be followed throughout this paper but the starred equations will not always be written out for the sake of brevity.

The governing equations $(2.3)$ and $\left(2.3^{*}\right)$ are solved subject to the auxiliary conditions

$$
\begin{gathered}
\hat{\chi}_{i 2}=0, \quad \text { on } \quad x_{2}=h, \\
\hat{u}_{i}=u_{i}, \quad \hat{\chi}_{i 2}=\chi_{i 2}, \quad \text { on } x_{2}=0, \\
u_{i} \rightarrow 0, \quad \text { as } \quad x_{2} \rightarrow-\infty .
\end{gathered}
$$

Following Cai and $\mathrm{Fu}$ [35], we may write the solution for the half-space in the form

$$
u_{1}=\left(A_{1} \mathrm{e}^{k s_{1} x_{2}}+A_{2} \mathrm{e}^{k s_{2} x_{2}}\right) \mathrm{e}^{i k x_{1}}, \quad u_{2}=\left(B_{1} \mathrm{e}^{k s_{1} x_{2}}+B_{2} \mathrm{e}^{k s_{2} x_{2}}\right) \mathrm{e}^{i k x_{1}},
$$

where $k$ is the wave number,

$$
B_{j}=\frac{i A_{j}\left(\gamma_{21} s_{j}^{2}-\alpha_{11}\right)}{\delta_{12} s_{j}}, j=1,2,
$$

$A_{1}, A_{2}$ are disposable constants, and $s_{1}$ and $s_{2}$ are the two roots of

$$
\alpha_{22} \gamma_{21} s^{4}-\left(\alpha_{11} \alpha_{22}+\gamma_{12} \gamma_{21}-\delta_{12}^{2}\right) s^{2}+\alpha_{11} \gamma_{12}=0
$$

that have positive real parts (so that $u_{i} \rightarrow 0$ as $x_{2} \rightarrow-\infty$ ). The constants $\alpha_{11}, \alpha_{22}$, $\gamma_{12}, \gamma_{21}, \delta_{12}$ are defined by

$$
\alpha_{11}=\mathcal{A}_{1111}^{1}, \quad \alpha_{22}=\mathcal{A}_{2222}^{1}, \quad \gamma_{12}=\mathcal{A}_{1212}^{1}, \quad \gamma_{21}=\mathcal{A}_{2121}^{1}, \quad \delta_{12}=\mathcal{A}_{1122}^{1}+\mathcal{A}_{1221}^{1} .
$$

For the coating the solution is not required to decay and so we have

$$
\hat{u}_{1}=\sum_{j=1}^{4} \hat{A}_{j} \mathrm{e}^{k \hat{s}_{j} x_{2}} \mathrm{e}^{i k x_{1}}, \quad \hat{u}_{2}=\sum_{j=1}^{4} \hat{B}_{j} \mathrm{e}^{k \hat{s}_{j} x_{2}} \mathrm{e}^{i k x_{1}}
$$




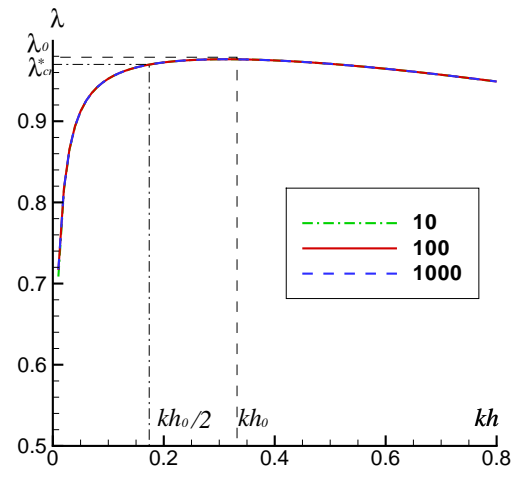

(a)

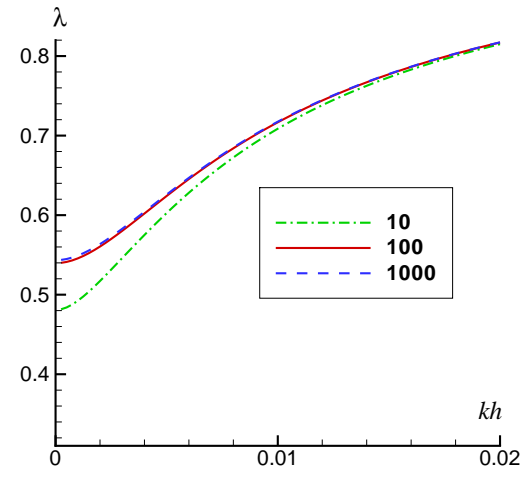

(b)

Figure 1: Bifurcation curves showing dependence of $\lambda$ on $k h$ for different $J_{m}$ in the case that both film and substrate are composed of Gent material. (b) is a blow-up of (a) for small values of $k h$.

where

$$
\hat{B}_{j}=\frac{i \hat{A}_{j}\left(\hat{\gamma}_{21} \hat{s}_{j}^{2}-\hat{\alpha}_{11}\right)}{\hat{\delta}_{12} \hat{s}_{j}}, \quad j=1,2,3,4,
$$

$\hat{A}_{1}, \hat{A}_{2}, \hat{A}_{3}, \hat{A}_{4}$ are disposable constants, $\hat{s}_{1}$ and $\hat{s}_{2}$ are the two roots of $\left(2.10^{*}\right)$ that have positive real parts, $\hat{s}_{3}=-\hat{s}_{1}, \hat{s}_{4}=-\hat{s}_{2}$, and the constants $\hat{\alpha}_{11}, \hat{\alpha}_{22}, \hat{\gamma}_{12}, \hat{\gamma}_{21}, \hat{\delta}_{12}$ are defined by $\left(2.11^{*}\right)$.

On substituting the above general solutions (2.8) and (2.12) into the auxiliary conditions (2.5) and (2.6), we obtain six linear homogeneous equations for the six constants $A_{1}, A_{2}, \hat{A}_{1}, \hat{A}_{2}, \hat{A}_{3}$ and $\hat{A}_{4}$. The six equations have a non-trivial solution if and only if the determinant of the coefficient matrix is zero, yielding a bifurcation condition in the form

$$
\Omega(\lambda, k h)=0,
$$

where $\lambda$ is the principal stretch in the $x_{1}$-direction. Note that the principal stretch in the $x_{2}$-direction is determined by solving the equation $\bar{\sigma}_{2}=0$ where $\bar{\sigma}_{2}$ is the Cauchy stress in the $x_{2}$-direction associated with the deformation $B_{0} \rightarrow B_{e}$. Equation (2.14) is easily solved with the aid of the symbolic manipulation package Mathematica [48]

To analyze the effect of material constitution, we shall focus on the case when the thin layer and half-space are both described by the incompressible Gent material model with the same values of $J_{m}$ but different shear moduli. This is achieved by taking the limits $\nu \rightarrow 1 / 2, \hat{\nu} \rightarrow 1 / 2$ in our numerical calculations. The relative stiffness of the film and half-space is characterized by the ratio $r=\hat{\mu} / \mu$. It is found sufficient to approximate the incompressible limit by taking $\hat{\nu}=\nu=0.4999$. For instance, in the case of $J_{m}=1000$ (so that the materials are almost neo-Hookean) and $r=10$, the critical principal stretch given by our nearly-incompressible approximation is 0.97635667 , whereas the exact bifurcation condition given by [35], which is valid for neo-Hookean materials, would give a critical principal stretch equal to 0.97635785 . 
In Fig. 1 we have shown the bifurcation curves for $r=100$ and $J_{m}=10,100$, 1000 , respectively. It can be seen that, for most values of $k h$, the variation in the critical stretch with respect to $J_{m}$ is very small (see Fig. 1(a)). But in the region where $k h$ is small, the critical stretch decreases rapidly as $J_{m}$ decreases (see the blow-up in Fig. 1(b)).

It is seen in Fig.1 that for the given values of $r$ and $J_{m}$, each bifurcation curve has a maximum for $\lambda$ which is denoted by $\lambda_{0}$. The corresponding value of $k h$ is denoted by $k_{0} h$ which gives the mode number $k_{0}$ of the bifurcation mode. As in Cai and Fu [35], when $r$ is much larger than 1, we may again derive the asymptotic expansions

$$
\lambda_{0}=1-\frac{1}{4}(3 / r)^{2 / 3}+\cdots, \quad k_{0} h=(3 / r)^{1 / 3}+\cdots .
$$

Thus, the leading order terms of $\lambda_{0}$ and $k_{0} h$ for Gent materials are independent of the material constant $J_{m}$ and are the same as for neo-Hookean materials [35]. It is found, however, that the second-order terms are dependent on $J_{m}$, but they are not written out here for the sake of brevity.

We also observe that on each bifurcation curve, there exists a point $\left(k_{0} h / 2, \lambda_{\mathrm{cr}}^{*}\right)$ which corresponds to a bifurcation mode that has twice the period of the primary buckling mode. This bifurcation mode will not be observable since the associated stretch value $\lambda_{\text {cr }}^{*}$ is smaller than $\lambda_{0}$, but it will feature in our analysis of secondary bifurcations.

To analyze the effect of compressibility, we assume that the thin-layer and halfspace are both composed of compressible neo-Hookean materials. This case is achieved by taking the limit $J_{m} \rightarrow \infty$, and is selected so as to compare with the case of an incompressible neo-Hookean material that has been much studied in the literature. The relative stiffness of the layer and half-space is still characterized by the magnitude of $r=\hat{\mu} / \mu$, but Poisson's ratio is assumed to be the same in both the film and substrate. In Fig. 2 we have shown the bifurcation curves for $r=100$ and $\nu=0.4$, $0.45,0.4999$, respectively. It can be seen that, as $\nu$ is varied, the changes in the critical stretch are also quite small.

When $r$ is much larger than 1 , we find that $\lambda_{0}$ and $k_{0} h$ have the asymptotic expansions

$$
\lambda_{0}=1-\frac{1}{4}\left(\frac{12(1-\nu)^{2}}{(3-4 \nu) r}\right)^{2 / 3}+\cdots, \quad k_{0} h=\left(\frac{12(1-\nu)^{2}}{(3-4 \nu) r}\right)^{1 / 3}+\cdots .
$$

The leading order terms of $\lambda_{0}-1$ and $k_{0} h$ are now dependent on Poisson's ratio. As expected, in the limit $\nu \rightarrow 1 / 2$, the leading-order terms in the above expansions reduce to those in $(2.15)$.

To analyze the effect of a pre-stretch on the critical stretch, we consider the case when both the layer and half-space are composed of different incompressible neoHookean materials (which is again approached by taking the appropriate limit as discussed earlier), but the substrate is subject to a pre-stretch. In this case, we can solve the bifurcation condition by simply letting the film and substrate have different final stretches, $\lambda_{f}$ and $\lambda_{s}$ say. 

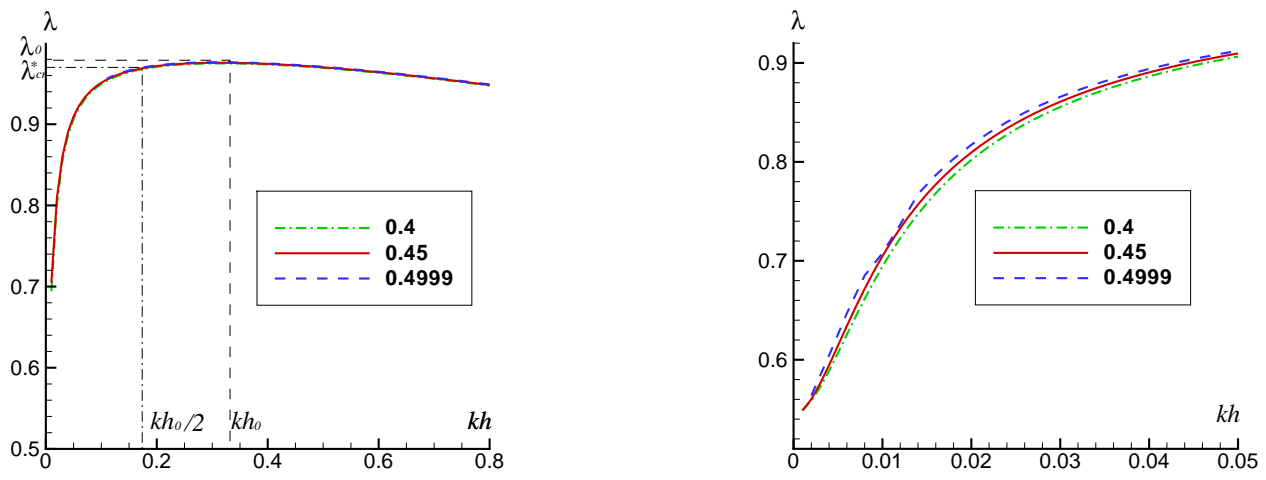

Figure 2: Bifurcation curves showing dependence of $\lambda$ on $k h$ for different Poisson's ratio in the case both film and substrate are composed of compressible neo-Hookean material. (b) is a blow-up of (a) for small values of $k h$.
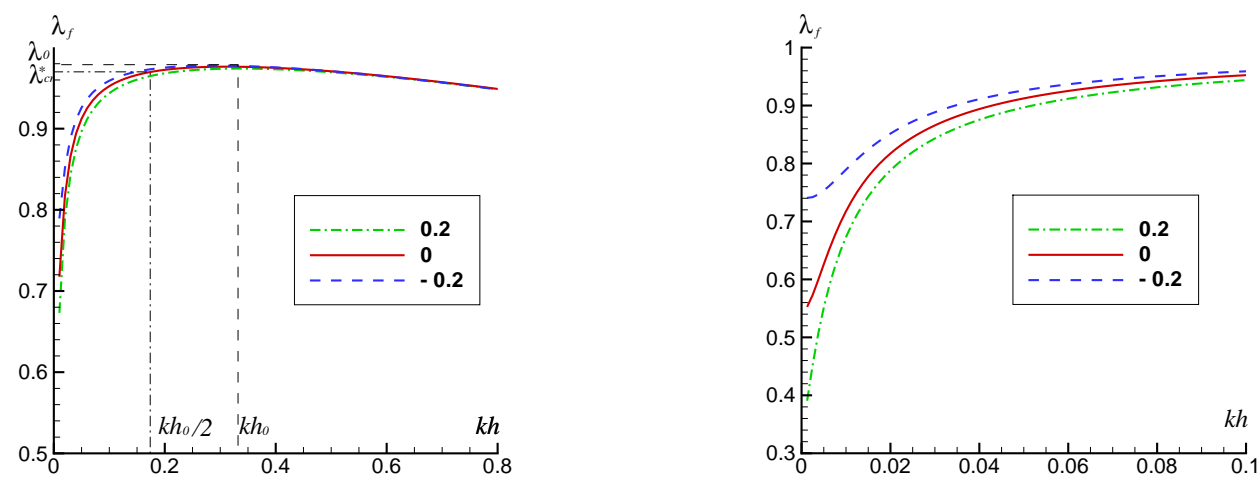

Figure 3: Bifurcation curves showing dependence of $\lambda$ on $k h$ for different pre-strain in substrate in the case both film and substrate are composed of neo-Hookean material. (b) is a blow-up of (a) for small values of $k h$.

In Fig. 3 we have shown the bifurcation curves for $r=100$ and $\Delta \equiv \lambda_{s}-\lambda_{f}=$ $-0.2,0,0.2$, respectively. It can be seen that allowing for a pre-stretch in the substrate has a much more pronounced effect on the critical stretch than varying the Poisson's ratio or the constant $J_{m}$.

When $r$ is much larger than 1 and the substrate is pre-stretched, the following asymptotic expressions may be derived:

$$
\lambda_{0}=1-\frac{1}{4}\left(\frac{6+6 \Delta+3 \Delta^{2}}{2 r}\right)^{2 / 3}+\cdots, \quad k_{0} h=\left(\frac{6+6 \Delta+3 \Delta^{2}}{2 r}\right)^{1 / 3}+\cdots .
$$

It is seen that the leading-order terms are now dependent on the pre-stretch. As expected, in the limit $\Delta \rightarrow 0$, these leading-order terms reduce to their counterparts in $(2.16)$. 


\section{Post-buckling solutions associated with the first bifurcation}

Solving the matrix equation that led to the bifurcation condition (2.14) will determine five of the six constants $A_{1}, A_{2}, \hat{A}_{1}, \ldots, \hat{A}_{4}$. As a result, the linear solution is determined to within a single multiplicative constant. This undetermined constant is determined in this section as part of the post-buckling solution using a weakly nonlinear perturbation procedure.

Anticipating that the bifurcation is super-critical, we write

$$
\lambda=\lambda_{0}-\epsilon^{2}
$$

where $\lambda_{0}$ is the critical stretch as defined in the previous section and $\epsilon$ is a positive small parameter.

For the analysis in the rest of this paper, it is convenient to view all dependent variables as functions of the coordinates in the critical configuration. To avoid introducing extra notations, these coordinates are still denoted by $x_{i}$ and the associated deformation gradient is still denoted by $\overline{\boldsymbol{F}}$. Thus, we now have

$$
\overline{\boldsymbol{F}}=\operatorname{diag}\left\{\lambda_{0}, \lambda_{20}\right\},
$$

where $\lambda_{20}$ is the value of $\lambda_{2}$, the principal stretch in the $x_{2}$-direction, when $\lambda_{1}$ takes its critical value $\lambda_{0}$. The definition (2.2) is still valid but the $\overline{\boldsymbol{F}}$ is now understood to be given by (3.2). To obtain the revised expression for $\chi_{i j}$, we first note that the expression (2.1) can now be rewritten as

$$
\begin{aligned}
& \tilde{x}_{1}=\lambda X_{1}+u_{1}(\boldsymbol{x})=\lambda \lambda_{0}^{-1} x_{1}+u_{1}(\boldsymbol{x})=x_{1}+w_{1}(\boldsymbol{x}), \\
& \tilde{x}_{2}=\lambda_{2} X_{2}+u_{2}(\boldsymbol{x})=\lambda_{2} \lambda_{20}^{-1} x_{2}+u_{2}(\boldsymbol{x})=x_{2}+w_{2}(\boldsymbol{x}),
\end{aligned}
$$

where

$$
w_{1}=\left(\lambda \lambda_{0}^{-1}-1\right) x_{1}+u_{1}(\boldsymbol{x}), \quad w_{2}=\left(\lambda_{2} \lambda_{20}^{-1}-1\right) x_{2}+u_{2}(\boldsymbol{x}) .
$$

The nonlinear equilibrium equations again take the simple form $(2.3)_{2}$, but now the constitutive equation (2.4) is replaced by

$$
\chi_{i j}=\mathcal{A}_{j i l k}^{1} w_{k, l}+\frac{1}{2} \mathcal{A}_{j i l k n m}^{2} w_{m, n} w_{k, l}+\frac{1}{6} \mathcal{A}_{j i l k n m q p}^{3} w_{m, n} w_{p, q} w_{k, l}+\cdots,
$$

where $\mathcal{A}^{2}$ and $\mathcal{A}^{3}$ are the second- and third-order tensors of instantaneous elastic moduli defined by

$$
\begin{gathered}
\mathcal{A}_{j i l k n m}^{2}=\left.\bar{J}^{-1} \bar{F}_{j A} \bar{F}_{l B} \bar{F}_{n C} \frac{\partial^{3} W}{\partial F_{i A} \partial F_{k B} \partial F_{m C}}\right|_{\mathbf{F}=\overline{\mathbf{F}}}, \\
\mathcal{A}_{\text {jilknmqp }}^{3}=\left.\bar{J}^{-1} \bar{F}_{j A} \bar{F}_{l B} \bar{F}_{n C} \bar{F}_{q E} \frac{\partial^{4} W}{\partial F_{i A} \partial F_{k B} \partial F_{m C} \partial F_{p E}}\right|_{\mathbf{F}=\overline{\mathbf{F}}} .
\end{gathered}
$$

Higher-order elastic moduli are defined in a similar manner. 
We look for an asymptotic solution of the form

$$
\begin{aligned}
& w_{j}=\epsilon w_{j}^{(1)}\left(x_{1}, x_{2}\right)+\epsilon^{2} w_{j}^{(2)}\left(x_{1}, x_{2}\right)+\epsilon^{3} w_{j}^{(3)}\left(x_{1}, x_{2}\right)+\cdots, \\
& \hat{w}_{j}=\epsilon \hat{w}_{j}^{(1)}\left(x_{1}, x_{2}\right)+\epsilon^{2} \hat{w}_{j}^{(2)}\left(x_{1}, x_{2}\right)+\epsilon^{3} \hat{w}_{j}^{(3)}\left(x_{1}, x_{2}\right)+\cdots,
\end{aligned}
$$

where the leading order solution is written in the form

$$
\left\{w_{j}^{(1)}, \hat{w}_{j}^{(1)}\right\}=A\left\{W_{j 1}^{(1)}\left(x_{2}\right), \hat{W}_{j 1}^{(1)}\left(x_{2}\right)\right\} E+\text { c.c. }, \quad E=\mathrm{e}^{i x_{1}} .
$$

In the last expression $A$ is the undetermined constant mentioned earlier, the shape functions $W_{j 1}^{(1)}\left(x_{2}\right)$ and $\hat{W}_{j 1}^{(1)}\left(x_{2}\right)$ are given by the linear analysis presented in the previous section, and the 'c.c.' denotes the complex conjugate of the preceding term. The expression for $E$ indicates that we have chosen the mode number of the critical mode to be unity. This means that we have used the inverse of the actual critical mode number $k_{0}$ as the length unit. Thus, for instance, the $h$ in the subsequent analysis is the actual layer thickness (in the critical configuration) multiplied by $k_{0}$.

The boundary-value problems satisfied by the higher order terms in the perturbation solution (3.5) can be derived by equating the coefficients of like powers of $\epsilon$ in the equilibrium equations and the auxiliary conditions $(2.5)-(2.7)$. It can be deduced that the second- and third-order solutions for the half-space must necessarily take the form

$$
\begin{gathered}
w_{j}^{(2)}=A \bar{A} W_{j 0}^{(2)}\left(x_{2}\right)+A^{2} W_{j 2}^{(2)}\left(x_{2}\right) E^{2}+\text { c.c. }, \\
w_{j}^{(3)}=B W_{j 1}^{(1)}\left(x_{2}\right) E+A^{3} W_{j 3}^{(3)}\left(x_{2}\right) E^{3}+\text { c.c. },
\end{gathered}
$$

where the constant $B$ and all the functions of $x_{2}$ appearing on the right hand sides are to be determined. Similar expressions can be written down for the thin layer. It is observed that the expression for $w_{i}^{(2)}$ only contains even powers of $E$ and $\bar{E}$ whereas $w_{i}^{(3)}$ only contains odd powers of $E$ and $\bar{E}$. This pattern can be generalized to higher-order solutions. Thus, for instance, $w_{i}^{(n)}$ with $n$ odd only contains terms proportional to $E^{n}, E^{n-2}, E^{n-4}, \ldots, E$, and their complex conjugates.

On substituting (3.7) and $\left(3.7^{*}\right)$ into the appropriate equilibrium equations and auxiliary conditions, and then equating the coefficients of $E^{0}$ and $E^{2}$, we obtain two sets of boundary value problems for the mean field and second harmonic at order $O\left(\epsilon^{2}\right)$. The mean field can be determined easily, but for the second harmonic it is found by elimination that $W_{22}^{(2)}\left(x_{2}\right)$ and $\hat{W}_{22}^{(2)}\left(x_{2}\right)$ each satisfies an inhomogeneous fourth-order differential equation of the form

$$
a_{4} W^{\prime \prime \prime \prime}\left(x_{2}\right)+a_{2} W^{\prime \prime}\left(x_{2}\right)+a_{0} W\left(x_{2}\right)=f\left(x_{2}\right),
$$

where $a_{0}, a_{2}, a_{4}$ are constants, and $f\left(x_{2}\right)$ is a known function. Although with the coefficients and $f\left(x_{2}\right)$ known this equation can be solved using the command DSolve in Mathematica [48] directly, underflow occurs when both exponentially growing and decaying terms are present in a single solution (as is the case for the layer). This difficulty is overcome by solving (3.9) with unknown coefficients first, and then replacing 
them afterwards with their numerical values. For the substrate, this underflow problem does not arise, but to avoid having to distinguish the decaying terms from the growing terms, the solution of (3.9) is obtained using the procedure outlined in [41]. This same procedure is also applied to find the third-order solution (3.8) and all the other higher-order solutions. In solving the problem for $W_{21}^{(3)}\left(x_{2}\right)$, obtained by equating the coefficients of $E$ at order $\epsilon^{3}$, a solvability condition needs to be imposed, and it is from this solvability condition that we obtain an amplitude equation for $A$ in the form

$$
A+c_{1} A^{2} \bar{A}=0
$$

where $c_{1}$ is a real constant that depends on the material properties of the bilayer structure. The non-trivial solution of (3.10) is given by

$$
|A|=\sqrt{-1 / c_{1}} \text {. }
$$

However, at this stage the $B$ appearing in the third-order solution (3.8) is still undetermined; it can only be determined from a solvability condition at order $\epsilon^{5}$.

As explained in [41], the amplitude $B$ can be determined using the virtual work method [49] without having to actually solve the problem at order $\epsilon^{5}$. The same method can also be applied to derive the amplitude equation (3.10) as a useful check although the problem at order $\epsilon^{3}$ has to be solved anyway because its solution is needed at orders $\epsilon^{4}$ and $\epsilon^{5}$. Suppose that substituting (3.5) into (3.4) yields an expression of the form

$$
\chi_{i j}=\epsilon \mathcal{A}_{j i l k}^{1} w_{k, l}^{(1)}+\sum_{\alpha=2}^{\infty} \epsilon^{\alpha}\left[\mathcal{A}_{j i l k}^{1} w_{k, l}^{(\alpha)}+R_{i j}^{(\alpha)}\right],
$$

where the term $R_{i j}^{(\alpha)}$ only involves solutions up to and including $\boldsymbol{w}^{(\alpha-1)}$. Then the virtual work method would yield the identity

$$
\begin{aligned}
& \int_{0}^{k} \int_{0}^{2 \pi} \hat{w}_{i}^{(0)} \hat{R}_{i j, j}^{(\alpha)} d x_{1} x_{2}+\int_{-\infty}^{0} \int_{0}^{2 \pi} w_{i}^{(0)} R_{i j, j}^{(\alpha)} d x_{1} x_{2} \\
= & \left.\int_{0}^{2 \pi} \hat{w}_{i}^{(0)} \hat{R}_{i 2}^{(\alpha)}\right|_{x_{2}=h} d x_{1}-\left.\int_{0}^{2 \pi} w_{i}^{(0)}\left(\hat{R}_{i 2}^{(\alpha)}-R_{i 2}^{(\alpha)}\right)\right|_{x_{2}=0} d x_{1},
\end{aligned}
$$

where

$$
\left\{w_{j}^{(0)}, \hat{w}_{j}^{(0)}\right\}=\left\{\hat{W}_{j 1}^{(1)}\left(x_{2}\right), \overline{\hat{W}}_{j 1}^{(1)}\left(x_{2}\right)\right\} \bar{E},
$$

and an overbar in (3.14) signifies complex conjugation. In view of the presence of $\bar{E}$ in (3.14), only those terms in $R_{i j}^{(\alpha)}$ that are proportional to $E$ will survive the integrations in (3.13). Equation (3.13) is effectively the solvability condition satisfied by the amplitude of the terms in $\hat{\boldsymbol{u}}^{(\alpha)}$ and $\boldsymbol{u}^{(\alpha)}$ that are proportional to $E$. We emphasize, however, that the use of the above virtual work method is not essential; the more elementary way of imposing the solvability condition will serve the same purpose although it will take longer time on Mathematica, especially at higher orders. 
The non-trivial solution (3.11) can only exist if the nonlinear coefficient $c_{1}$ is negative. Based on what is known for neo-Hookean material models, we expect that this is the case when $r$ is sufficiently large, and as $r$ is gradually reduced there exists a critical value of $r$ at which $c_{1}$ vanishes. Below this critical value, the bifurcation become subcritical and sensitive to imperfections. For Gent materials, the critical modulus ratio depends on the value of the stretchability parameter $J_{m}$. For $J_{m}=10$, 100 and 1000, the critical value of $r$ is equal to $1.89,1.77$ and 1.75 , respectively.

For compressible neo-Hookean materials, the critical value of $r$ depends on Poisson's ratio $\nu$. For $\nu=0.4,0.45$ and 0.4999 , the critical value of $r$ is found to be 2.11, 1.92 and 1.75 , respectively.

To conclude this section, we remark that in view of the fact that the origin in the $x_{1}$-direction can be arbitrarily chosen and the $W_{11}^{(1)}\left(x_{2}\right)$ and $W_{21}^{(1)}\left(x_{2}\right)$ in (3.6) are real and pure imaginary, respectively (see (2.8) and (2.9)), we may, without loss of generality, take $A$ to be real. Equation (3.6) can then be replaced by

$$
w_{1}^{(1)}=A W_{11}^{(1)}\left(x_{2}\right)(E+\bar{E}), \quad w_{2}^{(1)}=A W_{21}^{(1)}\left(x_{2}\right)(E-\bar{E}),
$$

and $\left(3.15^{*}\right)$. These expressions will be adopted in the following section.

\section{Period-doubling secondary bifurcation}

To determine the critical value of $\lambda$ at which a period-doubling secondary bifurcation can occur, we superimpose on $B_{t}$ a further infinitesimal incremental displacement $\mathbf{v}(\boldsymbol{x})$. If $\boldsymbol{W}$ and $\boldsymbol{V}$ are used to denote the tensors with components $w_{i, j}$ and $v_{i, j}$, respectively, with $w_{i}$ defined by (3.3), then the deformation gradient from $B_{0}$ to the final perturbed configuration is given by

$$
(\boldsymbol{I}+\boldsymbol{W}+\boldsymbol{V}) \overline{\boldsymbol{F}} .
$$

The appropriate incremental stress tensor is now defined by

$$
\chi_{i j}=\bar{J}^{-1}\left(S_{A i}-\tilde{S}_{A i}\right) \bar{F}_{j A},
$$

where $\overline{\boldsymbol{F}}$ is again given by (3.2) and $\tilde{S}_{A i}$ is the nominal stress associated with the deformation $B_{0} \rightarrow B_{t}$ with deformation gradient $\tilde{\boldsymbol{F}} \equiv(\boldsymbol{I}+\boldsymbol{W}) \overline{\boldsymbol{F}}$. To avoid introducing extra notation we have used the same notation $\chi_{i j}$ to mean the right hand side of (4.1) since the $\chi_{i j}$ in the previous section will never appear in the subsequent analysis again. In our previous paper [41], the incremental stress tensor was defined by $\chi_{i j}=\tilde{J}^{-1}\left(S_{A i}-\tilde{S}_{A i}\right) \tilde{F}_{j A}$. This was appropriate when $\mathbf{v}$ was viewed as a vector function of $\tilde{\boldsymbol{x}}$, but in the subsequent analysis the variable substitution $\tilde{\boldsymbol{x}} \rightarrow \boldsymbol{x}$ had to be employed to simplify analysis. We now realize that (4.1) is a much more convenient choice.

On expanding (4.1) around $\boldsymbol{F}=\tilde{\boldsymbol{F}}$, we obtain, to leading order,

$$
\chi_{i j}=\mathcal{A}_{j i l k} v_{k, l}, \quad \text { where } \mathcal{A}_{j i l k}=\left.\bar{J}^{-1} \bar{F}_{j A} \bar{F}_{l B} \frac{\partial^{2} W}{\partial F_{i A} \partial F_{k B}}\right|_{\boldsymbol{F}=\tilde{\boldsymbol{F}}} .
$$


The incremental equilibrium equation is obviously $\chi_{i j, j}=0$, which is to be solved subject to the auxiliary conditions

$$
\begin{gathered}
\hat{\chi}_{i 2}=0, \quad \text { on } x_{2}=h, \\
\hat{v}_{i}=v_{i}, \quad \hat{\chi}_{i 2}=\chi_{i 2}, \quad \text { on } x_{2}=0, \\
v_{i} \rightarrow 0, \quad \text { as } \quad x_{2} \rightarrow-\infty,
\end{gathered}
$$

where $\hat{\chi}_{i j}$ is given by $\left(4.2^{*}\right)$. Suppose the solution for the incremental problem have twice the period of the primary mode. We can then expand the critical stretch in the form

$$
\lambda_{0}=\lambda_{\mathrm{cr}}^{*}+\epsilon \lambda^{(1)}+\epsilon^{2} \lambda^{(2)}+\epsilon^{3} \lambda^{(3)}+\cdots,
$$

where $\lambda_{\mathrm{cr}}^{*}$ is the critical stretch at which a mode with mode number $1 / 2$ may bifurcate from the uniformly deformed state $B_{e}$ (see Fig. 1), $\lambda^{(1)}, \lambda^{(2)}, \lambda^{(3)}, \ldots$ are constants to be determined. As a result, the $\overline{\boldsymbol{F}}$ given by (3.2) must also be expanded. This expansion is crucial since it ensures that the leading-order problem has the solution with mode number $1 / 2$ and the incremental solution can be obtained by successive approximations.

Correspondingly, $\mathcal{A}_{\text {jilk }}$ can be expanded in terms of $\epsilon$ as

$$
\mathcal{A}_{j i l k}=\mathcal{A}_{0 j i l k}+\epsilon \mathcal{A}_{1 j i l k}+\epsilon^{2} \mathcal{A}_{2 j i l k}+\epsilon^{3} \mathcal{A}_{3 j i l k}+\cdots,
$$

where $\mathcal{A}_{0 j i l k}$ are constants, $\mathcal{A}_{1 j i l k}$ are functions of coordinates $x_{i}$ and parameter $\lambda^{(1)}$, $\mathcal{A}_{2 j i l k}$ are functions of coordinates $x_{i}$ and parameters $\lambda^{(1)}$ and $\lambda^{(2)}$, and so on. We note that in obtaining (4.7) only $\overline{\boldsymbol{F}}$ is re-expanded; the tensor $U$ was obtained from the previous section numerically and does not contain $\overline{\boldsymbol{F}}$ explicitly.

The incremental displacement can be expanded in the form

$$
v_{i}=v_{i}^{(0)}+\epsilon v_{i}^{(1)}+\epsilon^{2} v_{i}^{(2)}+\epsilon^{3} v_{i}^{(2)}+\cdots
$$

where $v_{i}^{(m)}(m=0,1, \ldots)$ are all functions of $x_{1}$ and $x_{2}$ and are to be determined at successive orders of approximations. By substituting this expansion into $\chi_{i j, j}=0$ and equating likes powers of $\epsilon$, we obtain the following sets of equations:

$O\left(\epsilon^{0}\right)$

$$
\mathcal{A}_{0 j i l k} v_{k, l}^{(0)}=0
$$

$O\left(\epsilon^{\alpha}\right), \quad \alpha \geq 1$

$$
\mathcal{A}_{0 j i l k} v_{k, l}^{(\alpha)}=H_{i}^{(\alpha)}\left(x_{1}, x_{2}: \lambda^{(\alpha)}\right)
$$

where the right hand side of (4.10) depends on the post buckling solution and incremental solutions up to and including order $\epsilon^{\alpha-1}$. By expanding the traction vector in the form

$$
\chi_{i 2}=T_{i}^{(0)}+\epsilon T_{i}^{(1)}+\epsilon^{2} T_{i}^{(2)}+\epsilon^{3} T_{i}^{(3)}+\cdots,
$$

we obtain the auxiliary conditions 
$O\left(\epsilon^{\alpha}\right), \quad \alpha=0,1,2,3, \ldots:$

$$
\begin{gathered}
T_{i}^{(\alpha)}=0, \quad \text { on } \quad x_{2}=k_{0}, \\
\hat{v}_{i}^{(\alpha)}=v_{i}^{(\alpha)}, \quad \hat{T}_{i}^{(\alpha)}=T_{i}^{(\alpha)}, \quad \text { on } x_{2}=0, \\
v_{i}^{(\alpha)} \rightarrow 0, \quad \text { as } x_{2} \rightarrow-\infty .
\end{gathered}
$$

The solution for (4.9) subjected to (4.12)-(4.14) with $\alpha=0$ is given by

$$
v_{i}^{(0)}=\mathrm{e}^{\mathrm{i} \phi} V_{i}^{(0)}\left(x_{2}\right) E^{1 / 2}+\text { c.c. }
$$

where $\phi$ is a constant satisfying $0 \leq \phi \leq \pi / 2, V_{i}^{(0)}$ can be obtained from the linear analysis of Section 2 by using the fact that $v_{i}^{(0)}$ is the bifurcation solution with mode number $1 / 2$ (when the scaled plate thickness is $k_{0} h$ ). In particular, $V_{1}^{(0)}\left(x_{2}\right)$ is real, whereas $V_{2}^{(0)}\left(x_{2}\right)$ is pure imaginary (see (see (2.8) and $\left.(2.9)\right)$ ). We also note that the above solution can be multiplied by an arbitrary real constant, but this is not necessary since we are solving a linearized eigenvalue problem.

Our numerical experimentation shows that a non-trivial solution can be found only if the $\phi$ in (4.15) is equal to 0 or $\pi / 2$. Furthermore, it can be shown that the solution with $\phi=\pi / 2$ can be obtained from the solution associated with $\phi=0$ by changing the signs of $\epsilon, \lambda^{(1)}, \lambda^{(3)}, \ldots$ Since the latter sign-changing does not alter the value of the right hand side of (4.6), the two bifurcation modes correspond to the same critical stretch. Thus, without loss of generality, we shall take $\phi=0$, and (4.15) then reduces to

$$
v_{1}^{(0)}=V_{1}^{(0)}\left(x_{2}\right)\left(E^{1 / 2}+\bar{E}^{1 / 2}\right), \quad v_{2}^{(0)}=V_{2}^{(0)}\left(x_{2}\right)\left(E^{1 / 2}-\bar{E}^{1 / 2}\right) .
$$

On substituting (4.15) into (4.10) for $\alpha=1$, the term on the right hand side becomes

$$
H_{i}^{(0)}\left(x_{1}, x_{2}\right)=R_{1}^{(0)}\left(x_{2} ; \lambda^{(1)}\right) E^{1 / 2}+R_{3}^{(0)}\left(x_{2}\right) E^{3 / 2}+\text { c.c. },
$$

where $R_{1}^{(0)}$ and $R_{3}^{(0)}$ are known functions. Thus the solution of (4.10) for $\alpha=1$ can be written in the form

$$
v_{i}^{(1)}=V_{i 1}^{(1)}\left(x_{2}\right) E^{1 / 2}+V_{i 3}^{(1)}\left(x_{2}\right) E^{3 / 2}+\text { c.c. } .
$$

By substituting (4.18) into (4.10) and equating the coefficient of $E^{1 / 2}$, we obtain two ordinary differential equations for $V_{11}^{(1)}\left(x_{2}\right)$ and $V_{21}^{(1)}\left(x_{2}\right)$. A similar operation applied to the thin layer yields two parallel equations for $\hat{V}_{11}^{(1)}\left(x_{2}\right)$ and $\hat{V}_{21}^{(1)}\left(x_{2}\right)$. These equations can be solved in the same manner as how the functions in (3.7) and (3.8) are found. The solution will contain a total of six disposable constants. On substituting this solution into the auxiliary conditions (4.12) and (4.13) we obtain a matrix equation of the form $M \boldsymbol{d}=\boldsymbol{f}$ where $\boldsymbol{d}$ is a 6 -vector formed from the six disposable constants, $\boldsymbol{f}$ is a known vector that contains $\lambda^{(1)}$, and $M$ is a $6 \times 6$ matrix whose determinant is zero. Imposition of an appropriate solvability condition then yields a linear equation for $\lambda^{(1)}$. In this way $\lambda^{(1)}$ is found and the $V_{i 1}^{(1)}\left(x_{2}\right)$ and $\hat{V}_{i 1}^{(1)}\left(x_{2}\right)$ are determined up to a multiplicative constant. 


\begin{tabular}{|c|c|c|}
\hline$J_{m}$ & $\lambda_{\mathrm{sec}}$ & $\epsilon_{\mathrm{sec}}$ \\
\hline 1 & 0.87581020 & 0.31698314 \\
2 & 0.85864093 & 0.34304869 \\
5 & 0.84474980 & 0.36275930 \\
10 & 0.83922600 & 0.37030394 \\
20 & 0.83625162 & 0.37430315 \\
100 & 0.83375778 & 0.37762340 \\
1000 & 0.83318177 & 0.37838613 \\
10000 & 0.83312386 & 0.37846274 \\
\hline
\end{tabular}

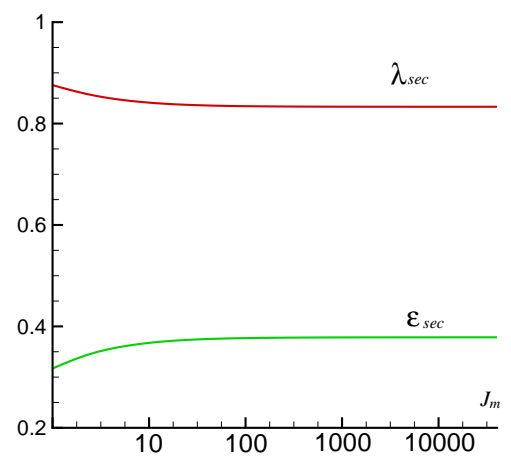

Figure 4: Period doubling secondary bifurcation critical stretch $\lambda_{\sec }$ and $\epsilon_{\sec }$ for Gent material.

In a similar manner, by substituting (4.18) into (4.10) and $\left(4.18^{*}\right)$ into $\left(4.10^{*}\right)$, and equating the coefficient of $E^{3 / 2}$, we obtain two ordinary differential equations for $V_{13}^{(1)}\left(x_{2}\right)$ and $V_{23}^{(1)}\left(x_{2}\right)$, and another two for $\hat{V}_{13}^{(1)}\left(x_{2}\right)$ and $\hat{V}_{23}^{(1)}\left(x_{2}\right)$. Solving these equations subject to the associated auxiliary conditions, we again obtain a matrix equation similar to the one discussed above, but now the coefficient matrix is not singular and a unique solution can be obtained. This completes the solution at order $\epsilon$.

By using the same procedure at higher orders we can obtain $\lambda^{(2)}, \lambda^{(3)}$ etc. When period-doubling secondary bifurcation can occur, equations (3.1) and (4.6) should be satisfied simultaneously. Truncating at $\epsilon^{3}$ and equating the right hand sides of these two equations, we obtain

$$
\lambda_{\mathrm{cr}}^{*}-\lambda_{0}+\epsilon \lambda^{(1)}+\epsilon^{2}\left(1+\lambda^{(2)}\right)+\epsilon^{3} \lambda^{(3)}=0 .
$$

This cubic equation for $\epsilon$ has at least one real root. It turns out that for all the cases that we have considered, it is the only real root which we denote by $\epsilon_{\mathrm{sec}}$. We note, however, that $\epsilon_{\mathrm{sec}}$ is allowed to be negative. As discussed in the two paragraphs below (4.15), when a solution with negative $\epsilon_{\mathrm{sec}}$ is found, another solution can be constructed by changing the signs of $\epsilon_{\mathrm{sec}}, \lambda^{(1)}, \lambda^{(3)}$ etc, and the two solutions correspond to the same critical stretch. The critical stretch for period-doubling secondary bifurcation is computed from

$$
\lambda_{\mathrm{sec}}=\lambda_{0}-\epsilon_{\mathrm{sec}}^{2}
$$

which is independent of the sign of $\epsilon_{\mathrm{sec}}$ as expected.

When the layer and substrate are both modelled as Gent materials with $\hat{\mu} / \mu=$ $100, \lambda_{\mathrm{sec}}$ and $\epsilon_{\mathrm{sec}}$ are dependent on $J_{m}$. This dependence is shown in Figure 4 . It can be seen that $\lambda_{\text {sec }}$ is a monotonically decreasing function of $J_{m}$, but is not too sensitive to changes in $J_{m}$ except when $J_{m}$ becomes smaller than 10. Numerical values of $\lambda_{\text {sec }}$ and $\epsilon_{\mathrm{sec}}$ for some selected values of $J_{m}$ are listed in a table in the same figure. As $J_{m} \rightarrow \infty, \lambda_{\text {sec }}$ tends to the value for neo-Hookean materials. 


\begin{tabular}{|c|c|c|}
\hline$v$ & $\lambda_{\mathrm{sec}}$ & $\epsilon_{\mathrm{sec}}$ \\
\hline 0.499 & 0.83317751 & 0.37837623 \\
0.45 & 0.83258148 & 0.37830343 \\
0.40 & 0.81740097 & 0.39689526 \\
0.35 & 0.76107512 & 0.46154106 \\
0.31 & 0.38827069 & 0.76493849 \\
0.20 & 0.3645871 & -0.77900054 \\
0.10 & 0.60784548 & -0.60147016 \\
0.00 & 0.69550493 & -0.52182921 \\
\hline
\end{tabular}

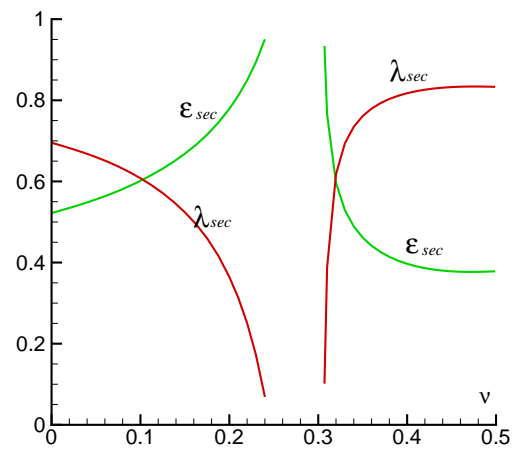

Figure 5: Period doubling secondary bifurcation critical stretch $\lambda_{\sec }$ and $\epsilon_{\sec }$ for compressible neoHookean material.

\begin{tabular}{|c|c|c|}
\hline$\Delta$ & $\lambda_{\text {sec }}$ & $\epsilon_{\text {sec }}$ \\
\hline-0.18 & 0.56124411 & 0.64525690 \\
-0.10 & 0.85437892 & 0.35043132 \\
-0.05 & 0.84816505 & 0.35866498 \\
0.00 & 0.83311743 & 0.37847125 \\
0.10 & 0.80009970 & 0.41854801 \\
0.24 & 0.78230530 & 0.43720932 \\
0.40 & 0.79095841 & 0.42434836 \\
0.80 & 0.81602003 & 0.38453531 \\
\hline
\end{tabular}

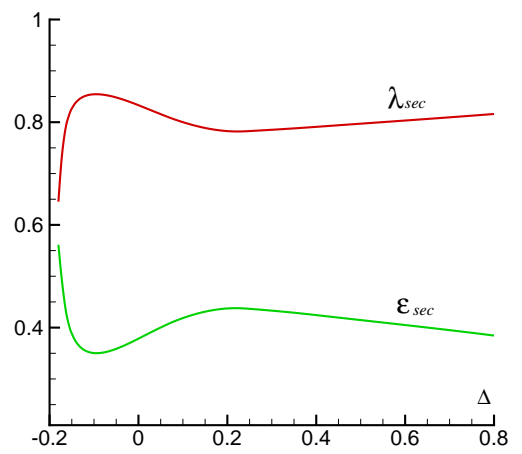

Figure 6: Period doubling secondary bifurcation critical stretch $\lambda_{\mathrm{sec}}$ and $\epsilon_{\mathrm{sec}}$ for incompressible neo-Hookean material with pre-strain in substrate.

When the layer and substrate are both modelled as compressible neo-Hookean materials with $\hat{\mu} / \mu=100, \lambda_{\text {sec }}$ and $\epsilon_{\text {sec }}$ are dependent on $\nu$, and their variations are shown in Figure 5 . It can be seen that $\lambda_{\text {sec }}$ is a monotonically increasing function of $\nu$. As $\nu \rightarrow 1 / 2, \lambda_{\mathrm{sec}}$ tends to the value for neo-Hookean materials. As $\nu$ approaches a threshold value approximately equal to 0.31 , the parameter $\epsilon_{\mathrm{sec}}$, which is meant to be sufficiently small, increases rapidly. Our asymptotic expansions, and hence our result for $\lambda_{\text {sec }}$, become invalid. We may then tentatively conclude that perioddoubling secondary bifurcation may become impossible or is not the preferred mode of bifurcation in this limit. This is discussed further in the concluding section.

Finally, we consider the case when the layer and substrate are both modelled as incompressible neo-Hookean materials with $\hat{\mu} / \mu=100$, but a pre-stretch is allowed in the substrate before the bilayer structure is compressed. The pre-stretch is characterized by $\Delta$, the mismatch of the stretch experienced by the substrate and the layer. The dependence of $\lambda_{\mathrm{sec}}$ and $\epsilon_{\mathrm{sec}}$ on $\Delta$ is shown in Figure 6 . It can be seen that 


\begin{tabular}{|c|c|c|}
\hline$v$ & $\lambda_{\mathrm{sec}}$ & $\epsilon_{\mathrm{sec}}$ \\
\hline 0.499 & 0.83272633 & 0.37897294 \\
0.45 & 0.83351358 & 0.37710920 \\
0.40 & 0.83228186 & 0.37774835 \\
0.35 & 0.82706322 & 0.38352410 \\
0.30 & 0.81712071 & 0.39517754 \\
0.20 & 0.77884362 & 0.43889470 \\
0.10 & 0.69465279 & 0.52436519 \\
0.00 & 0.45816966 & 0.71384357 \\
\hline
\end{tabular}

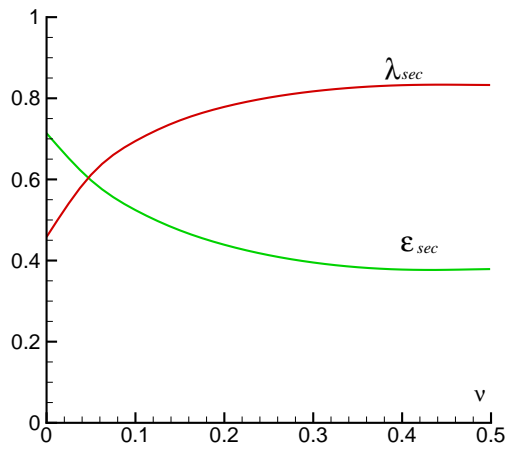

Figure 7: Period doubling secondary bifurcation critical stretch $\lambda_{\mathrm{sec}}$ and $\epsilon_{\mathrm{sec}}$ for compressible material (5.1).

the effects of pre-strain on $\lambda_{\mathrm{sec}}$ is not monotonic. When the pre-strain is negative, as its amplitude increases the critical compression decreases firstly and then increases after the pre-strain has reached a value approximately equal to $\Delta=-0.1$. When the pre-strain is positive, the critical compression increases first and then decreases after the pre-strain has reached a value approximately equal to $\Delta=0.24$. The monotonic behavior in a sufficiently small neighborhood of $\Delta=0$ is consistent with the experimental results given by Auguste et al. [50] and the numerical results given by Zhuo and Zhang [46], but the non-monotonic behavior outside this neighborhood was not considered by the latter authors.

\section{Discussion}

In this paper we have refined the methodology proposed in Fu and Cai [41] and used it to assess the effects of compressibility, a pre-stretch in the substrate, and material models on the critical stretch at which period-doubling secondary bifurcation occurs in a uniaxially compressed film-substrate bilayer structure. Our numerical results show that for a bilayer composed of incompressible materials, the constitutive model significantly affects the magnitude of the critical compression at which perioddoubling secondary bifurcation occurs. Comparing with the neo-Hookean model, the extensibility parameter $J_{m}$ in the Gent model plays an important role in the perioddoubling secondary bifurcation. It is found that the critical strain is a monotonically increasing function of $J_{m}$, achieving its maximum in the limit $J_{m} \rightarrow \infty$, that is when the layer and substrate are both modelled as neo-Hookean materials. The critical strain becomes as small as 0.12 when $J_{m}$ is equal to 1 .

A pre-strain in the substrate is another important factor affecting the critical compression at which period-doubling secondary bifurcation occurs. Numerical results show that when $\hat{\mu} / \mu=100$ this critical value attains a local maximum when the substrate is given a pre-strain of about $10 \%$, and a local minimum when the substrate is subject to a pre-strain of about $24 \%$. 
For compressible materials, Poisson's ratio plays a crucial role in period-doubling secondary bifurcations. When the layer and substrate are both composed of compressible neo-Hookean materials with the same Poisson's ratio, numerical results corresponding to the particular choice $\hat{\mu} / \mu=100$ show that the critical compression is an increasing function of Poisson's ratio $\nu$ with the incompressible limit recovered correctly when $\nu$ tends to 0.5 . Our calculations seem to indicate that there exists a critical value of Poisson's ratio, approximately equal to 0.307 , below which perioddoubling secondary bifurcation becomes impossible or gives way to other modes of bifurcations. To understand how this result depends on the constitutive model used, we have also considered the strain-energy function given by

$$
W=\frac{\mu}{2}\left(\bar{I}_{1}-3\right)+\frac{\mu^{*}}{2}(J-1)^{2},
$$

where $\bar{I}_{1}=I_{1} J^{-2 / 3}, \mu^{*}=2 \mu(1+\nu) /[3(1-2 \nu)]$. This is the compressible neo-Hookean material model built into the Abaqus software. In the case $\hat{\mu} / \mu=100$, the variations of $\lambda_{\mathrm{sec}}$ and $\epsilon_{\mathrm{sec}}$ on $\nu$ are shown in Figure 7 . It can be seen that now $\lambda_{\text {sec }}$ is not a monotonically increasing function of $\nu$. As $\nu$ increases, $\lambda_{\text {sec }}$ increases first and then decreases after a maximum arrived (the data in the accompanying table show this behaviour more clearly). As $\nu \rightarrow 1 / 2, \lambda_{\sec }$ tends to the value for neo-Hookean materials. In this case the asymptotic results are feasible for all values of $\nu$ although the critical strains are quite large for small values of $\nu$.

We have carried out some preliminary numerical simulations on Abaqus using both material models (1.1) and (5.1). As $\nu$ is decreased from its incompressible limit $1 / 2$, our asymptotic results for $\lambda_{\text {sec }}$ are initially very close to their numerical counterparts (with a relative error less than $5 \%$ ), but the two sets of results gradually diverge from each other. We believe that this might be due to the fact that for values of $\nu$ small enough, other secondary bifurcations may also be possible and may be preferred. To provide some evidence to this claim, we have also considered secondary bifurcations into a mode with a mode number $1 / N$, where $N$ is a rational number greater than unity but not equal to 2 . The leading-order solution (4.15) is replaced by

$$
v_{i}^{(0)}=V_{i}^{(0)}\left(x_{2}\right) E^{1 / N}+\text { c.c.. }
$$

From the interaction of this mode with the primary bifurcation mode (3.5), we deduce that solutions at the next two orders must necessarily take the form

$$
\begin{gathered}
v_{i}^{(1)}=V_{i 1}^{(1)}\left(x_{2}\right) E^{1-1 / N}+V_{i 3}^{(1)}\left(x_{2}\right) E^{1+1 / N}+\text { c.c. } \\
v_{i}^{(2)}=V_{i 1}^{(2)}\left(x_{2}\right) E^{1 / N}+V_{i 2}^{(2)}\left(x_{2}\right) E^{2-1 / N}+V_{i 3}^{(2)}\left(x_{2}\right) E^{2+1 / N}+\text { c.c. }
\end{gathered}
$$

respectively. Thus, no solvability condition is necessary for $v_{i}^{(1)}$, but is needed for $v_{i}^{(2)}$. From such considerations, we deduce that the principal stretch may be expanded as

$$
\lambda_{0}=\lambda_{\mathrm{cr}}^{*}+\epsilon^{2} \lambda^{(2)}+\epsilon^{4} \lambda^{(4)}+\ldots
$$

where $\lambda_{\mathrm{cr}}^{*}$ is the critical stretch at which a mode with mode number $1 / N$ may bifurcate from the uniformly deformed state $B_{e}$, and $\lambda^{(2)}, \lambda^{(4)}, \ldots$ are constants to be 


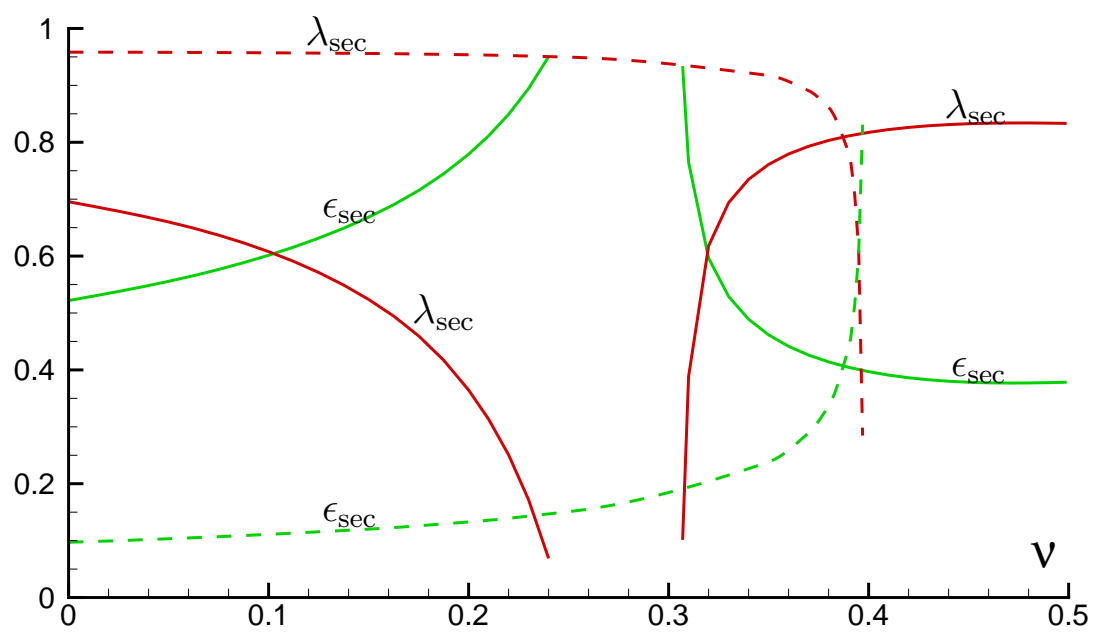

Figure 8: Asymptotic results for secondary bifurcations with $N=7 / 4$ (dashed lines). The solid lines are the same results as in Figure 5 and are included for comparison.

determined. Truncating at $\epsilon^{4}$ and equating the right hand side of (5.5) to $\lambda_{0}-\epsilon^{2}$, we obtain

$$
\lambda_{\mathrm{cr}}^{*}-\lambda_{0}+\epsilon^{2}\left(1+\lambda^{(2)}\right)+\epsilon^{4} \lambda^{(4)}=0 .
$$

From (5.6), we may find real roots of $\epsilon$. Denote the smallest positive root by $\epsilon_{\mathrm{sec}}$, then the critical stretch for the secondary bifurcation is computed with the use of $\lambda_{\mathrm{sec}}=\lambda_{0}-\epsilon_{\mathrm{sec}}^{2}$. By using the above procedure, we have tried various rational values of $N$ close to 2 and have indeed found solutions when $N=7 / 4$ when the strain-energy function (1.1) is used. The solutions are plotted together with the solutions for $N=2$ in Figure 8. It is seen that for $\nu$ less than 0.4 approximately, the mode with $N=7 / 4$ corresponds to lower values of the compression strain. Without theoretical guidance, such a secondary bifurcation mode might be difficult to detect in purely numerical simulations. From a dynamical system's point of view, secondary bifurcations with larger periods are always possible. This has indeed previously been demonstrated numerically by Budday et al [40] who showed that period-tripling, period-quadrupling, and period-quintupling secondary bifurcations are also possible although they occur at higher values of compressive strain in the specific context considered.

Since the solution behaviour as $\nu$ approaches 0.31 in Fig. 5 is similar to the solution behaviour as $\hat{\mu} / \mu$ approaches 5.8 found in [41] for the incompressible case, the above solutions found for $N=7 / 4$ prompted us to look for similar solutions when $\hat{\mu} / \mu$ satisfies $1.74<\hat{\mu} / \mu<5.8$ and when both the layer and substrate are incompressible. We confirm that secondary bifurcation solutions with $N=7 / 4$ do indeed exist in this parameter regime as well.

Finally, we observe that in our previous paper [41], the post-buckling solution 
is also assumed to be proportional to $\mathrm{e}^{i x_{1}}$ but the $x_{1}$ there is given by $x_{1}=\lambda X_{1}$ where $\lambda=\lambda_{0}-\epsilon^{2}$. Thus, measurement of period-doubling is with respect to the uniformly compressed configuration with $\lambda=\lambda_{0}-\epsilon^{2}$, whereas here it is with respect to the configuration with $\lambda=\lambda_{0}$. Equally, we could have assumed that all dependent variables are functions of $X_{A}$ and the post-buckling solution is proportional to $\mathrm{e}^{\mathrm{i} X_{1}}$. In this case measurement of period-doubling is then with respect to the initial uncompressed configuration. In the current paper, $\lambda_{0}$ and $\lambda_{\mathrm{cr}}^{*}$ are determined from

$$
\Omega\left(\lambda_{0}, k h\right)=0, \quad \text { and } \Omega\left(\lambda_{\mathrm{cr}}^{*}, \frac{1}{2} k h\right)=0,
$$

respectively; see (2.14). Denote the associated principal stretches in the $x_{2}$-direction by $g\left(\lambda_{0}\right)$ and $g\left(\lambda_{\mathrm{cr}}^{*}\right)$, respectively. If, for instance, period-doubling were measured in terms of $X_{A}$, then $\lambda_{0}$ and $\lambda_{\mathrm{cr}}^{*}$ would be determined by

$$
\hat{\Omega}\left(\lambda_{0}, K H\right)=0, \quad \text { and } \hat{\Omega}\left(\lambda_{\mathrm{cr}}^{*}, \frac{1}{2} K H\right)=0,
$$

where $K$ is the mode number such that the solution is proportional to $\mathrm{e}^{\mathrm{i} K X_{1}}$. Consistency of $(5.7)_{1}$ and $(5.8)_{1}$ requires

$$
\hat{\Omega}\left(\lambda_{0}, K H\right)=\Omega\left(\lambda_{0}, \frac{K}{\lambda_{0}} g\left(\lambda_{0}\right) H\right) .
$$

However, if $\hat{\Omega}$ were defined this way, $(5.7)_{2}$ and $(5.8)_{2}$ would in general be inconsistent. Thus, the three ways of measuring period-doubling give different results, but the differences are found to be insignificant. The Mathematica programme used to produce the results in this paper is freely available to any interested reader upon request.

Acknowledgment: This work was supported by the National Natural Science Foundation of China (grants 11372212 and 11672202), and by the National Basic Research Program of China (grant 2013CB035402). We thank Dr. Fan Xu and Mr. Lishuai Jin for carrying out some preliminary numerical simulations using Abaqus.

\section{References}

[1] H.-W. Chien, W.-H. Kuo, M.-J. Wang, S.-W. Tsai, and W.-B. Tsai. Tunable micropatterned substrates based on poly(dopamine) deposition via microcontact printing. Langmuir, 28:5775-5782, 2012.

[2] S G Lee, D Y Lee, H S Lim, D H Lee, S Lee, and K Cho. Switchable transparency and wetting of elastomeric smart windows. Adv. Mater., 22:5013C5017, 2010.

[3] T Ma, H Liang, G Chen, B Poon, H Jiang, and Yu H. Micro-strain sensing using wrinkled stiff thin films on soft substrates as tunable optical grating. Opt. Express, 21:11994-12001, 2013. 
[4] P Kim, Y Hu, J Alvarenga, M Kolle, Z Suo, and J Aizenberg. Rational design of mechano-responsive optical materials by fine tuning the evolution of straindependent wrinkling patterns. Adv. Opt. Mater., 1:381-388, 2013.

[5] E P Chan, E J Smith, R C Hayward, and A J Crosby. Surface wrinkles for smart adhesion. Adv. Mater., 20:711C716, 2008.

[6] S Yang, K Khare, and P C Lin. Harnessing surface wrinkle patterns in soft matter. Adv. Funct. Mater, 20:2550-2564, 2010.

[7] Z Q Zhang, T Zhang, Y W Zhang, K.-S. Kim, , and H J Gao. Strain-controlled switching of hierarchically wrinkled surfaces between superhydrophobicity and superhydrophilicity. Langmuir, 28:2753-2760, 2012.

[8] C M Stafford, C Harrison, K L Beers, A Karim, Amis E J, M R VanLandingham, H C Kim, W Volksen, R D Miller, and E E Simonyi. A buckling-based metrology for measuring the elastic moduli of polymeric thin films. Nat. Mater., 3:545-550, 2004 .

[9] E P Chan, K A Page, S H Im, D L Patton, R Huang, and C M Stafford. Viscoelastic properties of confined polymer films measured via thermal wrinkling. Soft Matter, 5:4638C4641, 2009.

[10] N Bowden, S Brittain, A G Evans, J W Hutchinson, and G M Whitesides. Spontaneous formation of ordered structures in thin films of metals supported on an elastomeric polymer. Nature, 393:146-149, 1998.

[11] N Bowden, W T S Huck, Paul K E, and G M Whitesides. The controlled formation of ordered, sinusoidal structures by plasma oxidation of an elastomeric polymer. Appl. Phys. Lett., 75:2557-2559, 1999.

[12] B Li, Y P Cao, X Q Feng, and H J Gao. Mechanics of morphological instabilities and surface wrinkling in soft materials: a review. Soft Matter, 8:5728-5745, 2012 .

[13] A Goriely. The mathematics and mechanics of biological growth. Springer, 2017.

[14] Q M Wang and X H Zhao. Beyond wrinkles: multimodal surface instabilities for multifunctional patterning. MRS Bulletin, 41:115-122, 2016.

[15] M A Holland, B Li, X Q Feng, and E Kuhl. Instabilities of soft films on compliant substrates. J. Mech. Phy. Solids, 98:350-365, 2017.

[16] D Bigoni, M Ortiz, and A Needleman. Effect of interfacial compliance on bifurcation of a layer bonded to a substrate. Int. J. Solids Struct., 34:4305-4326, 1997. 
[17] X Chen and J W Hutchinson. Herring bone buckling patterns of compressed thin films on compliant substrates. J. Appl. Mech., 71:597-603, 2004.

[18] Z Y Huang, W Hong, and Z Suo. Nonlinear analyses of wrinkles in a film bonded to a compliant substrate. J. Mech. Phys. Solids, 53:2101-2118, 2005.

[19] B Audoly and A Boudaoud. Buckling of a stiff film bound to a compliant substrate - part i: formulation, linear stability of cylindrical patterns, secondary bifurcations. J. Mech. Phys. Solids, 56:2401-2421, 2008.

[20] J Song, H Jiang, Z J Liu, D Y Khang, Y Huang, J A Rogers, C Lu, and C G Koh. Buckling of a stiff thin film on a compliant substrate in large deformation. Int. J. Solids Struct., 45:3107-3121, 2008.

[21] Y P Cao and J W Hutchinson. From wrinkles to creases in elastomers: the instability and imperfection-sensitivity of wrinkling. Proc. R. Soc. Lond. A, 468:94-115, 2012.

[22] Y P Cao, F Jia, X Q Feng, and S W Yu. Buckling and post-buckling of a stiff film resting on an elastic graded substrate. Int. J. Solids Struct., 49:1656-1664, 2012.

[23] J F Zang, X H Zhao, Y P Cao, and J W Hutchinson. Localized ridge wrinkling of stiff films on compliant substrates. J. Mech. Phys. Solids, 60:1265-1279, 2012.

[24] P Ciarletta and Y B Fu. A semi-analytical approach to biot instability in a growing layer: Strain gradient correction, weaklynon-linearanalysis and imperfection sensitivity. Int. J. Non-linear Mech., 75:38-45, 2015.

[25] F Xu and M Potier-Ferry. A multi-scale modeling framework for instabilities of film/substrate systems. J. Mech. Phys. Solids, 86:150-172, 2016.

[26] Z Chen, W Q Chen, and J Z Song. Buckling of a stiff thin film on an elastic graded compliant substrate. Proc. R. Soc. Lond., 473:20170410, 2017.

[27] F Xu and M Potier-Ferry. Quantitative predictions of diverse wrinkling patterns in film/substrate systems. Sci. Rep., 7:18081, 2017.

[28] Z Chen, X F Zhang, and J Z Song. Surface wrinkling of an elastic graded layer. Soft Matter, 14:8717-8723, 2018.

[29] L S Jin, Y Liu, and Z X Cai. Asymptotic solutions on the circumferential wrinkling of growing tubular tissues. Int. J. Eng. Sci, 128:31-43, 2018.

[30] F Jia, S P Pearce, and A Goriely. Curvature delays growth-induced wrinkling. Phys. Rev. E, 98:033003, 2018. 
[31] J J Sui, J B Chen, X X Zhang, G H Nie, and T Zhang. Symplectic analysis of wrinkles in elastic layers with graded stiffnesses. J. Appl. Mech., 86:011008, 2018.

[32] Y F Yang, H.-H. Dai, F Xu, and M Potier-Ferry. Pattern transition in a soft cylindrical shell. Phys. Rev. Lett., 120:215503, 2018.

[33] H Alawiye, E Kuhl, and A Goriely. Revisiting the wrinkling of elastic bilayers i: linear analysis. Phil. Tran. R. Soc. A, 377:20180076, 2019.

[34] L S Jin, Y Liu, and Z X Cai. Post-buckling analysis on growing bubular tissues: a semi-analytical approach and imperfection sensitivity. Int. J. Solids Struct., 162:121-134, 2019.

[35] Z X Cai and Y B Fu. On the imperfection sensitivity of a coated elastic halfspace. Proc. R. Soc. Lond. A, 455:3285-3309, 1999.

[36] J W Hutchinson. The role of nonlinar substrate elasticity in the wrinkling of thin films. Phil. Trans. R. Soc., A, 371:20120422, 2013.

[37] S Q Cai, D Y Chen, Z G Suo, and R C Hayward. Creasing instability of elastomer films. Soft Matter, 8:1301-1304, 2012.

[38] Y B Fu and P Ciarletta. Buckling of a coated elastic half-space when the coating and substrate have similar material properties. Proc. R. Soc. Lond. A, 471:20140979, 2014.

[39] J Liu and K Bertoldi. Bloch wave approach for the analysis of sequential bifurcations in bilayer structures. Proc. R. Soc. Lond. A, 471:20150493, 2015.

[40] S Budday, E Kuhl, and J W Hutchinson. Period-doubling and period-tripling in growing bilayered systems. Phil. Mag., 95:3208-3309, 2015.

[41] Y B Fu and Z X Cai. An asymptotic analysis of the period-doubling secondary bifurcation in a film/substrate bilayer. SIAM J. Appl. Math., 75:2381-2395, 2015.

[42] Y P Cao and J W Hutchinson. Wrinkling phenomena in neo-hookean film/substrate bilayers. ASME J. Appl. Mech., 79:031019, 2012.

[43] F Brau, H Vandeparre, A Sabbah, C Poulard, A Boudaoud, and P Damman. Multiple-length-scale elastic instability mimics parametric resonance of nonlinear oscillators. Nat. Phys, 7:56-60, 2011.

[44] Y Zhao, Y P Cao, W Hong, M K Wadee, and X Q Feng. Towards a quantitative understanding of period-doubling wrinkling patterns occurring in film/substrate bilayer systems. Proc. R. Soc. Lond. A, 471:20140695, 2015. 
[45] L J Zhuo and Y Zhang. The mode-coupling of a stiff film/compliant substrate system in the post-buckling range. Int. J. Solids Struct., 53:28-37, 2015.

[46] L J Zhuo and Y Zhang. From period-doubling to folding in stiff film/soft substrate system: The role of substrate nonlinearity. Int. J. Non-linear Mech., 76: $1-7,2015$.

[47] P Chadwick and R W Ogden. On the definition of elastic moduli. Arch. Ration. Mech. Anal., 44:41-53, 1971.

[48] S Wolfram. Mathematica: A System for Doing Mathematics by Computer (2nd Edn). Addison-Wesley, California, 1991.

[49] Y B Fu. Resonant-triad instability of a pre-stressed incompressible elastic plate. J. Elast., 41:13-37, 1995.

[50] A Auguste, L H Jin, Z G Suo, and R C Hayward. The role of substrate prestretch in post-wrinkling bifurcations. Soft Matter, 10:6520-6529, 2014. 